\title{
CODIFICADORES CONVOLUCIONAIS ABELIANOS
}

\author{
Jorge Pedraza Arpasi*e Reginaldo Palazzo Jr. ${ }^{\dagger}$ \\ DT/FEEC/UNICAMP \\ Caixa Postal 6101 \\ 13081-970 Campinas SP
}

Resumo - Neste trabalho são estabelecidas as condições necessárias e suficientes para a construção de codificadores convolucionais abelianos. Mostramos que tais codificadores são controláveis, completos e mínimos, esta última proposição via uma proposta de redução de estados, e tendo como subclasse os codificadores convolucionais elementares. Como consequência do fato de que os codificadores convolucionais abelianos controláveis, cuja seção de treliça é isomorfa a um grupo cíclico, não são controláveis, então propomos um algoritmo de construção de codificadores convolucionais abelianos tendo como grupo das entradas, de estados e de saídas, $k$-uplas, $(n-k)$-uplas e $n$-uplas binárias, respectivamente. Exemplos de codificadores convolucionais elementares e abelianos são apresentados.

Abstract - In this paper we establish the necessary and sufficient conditions for the construction of Abelian convolutional encoders. We show that such encoders are controllable, complete and minimal, the latter via a state reduction procedure, and having the elementary convolutional encoders as a subclass. As a consequence of the fact that the Abelian convolutional encoders whose trellis section is isomorphic to a cyclic group are noncontrollable, then an algorithm for the construction of controllable Abelian convolutional encoders whose trellis section is isomorphic to an Abelian group (not cyclic) is proposed having the group of input, the group of states, and the group of output binary $k$-tuples, $n-k$-tuples and $n$-tuples, respectively. Examples of elementary and Abelian convolutional encoders are presented.

Palavras Chaves : Máquinas, codificadores convolucionais elementares, codificadores convolucionais abelianos, controlabilidade.

\section{INTRODUÇÃO}

Se para cada $s \in S$ existe $g \in \Gamma(S)$ tal que $s=g\left(s_{0}\right)$, para algum $s_{0} \in S$, então dizemos que $\Gamma(S)$ atua transitivamente sobre $S$. O ponto $s_{0} \in S$ é denominado semente ou gerador de $S$. Note que podem existir vários geradores de $S$.

Para se obter códigos Euclidianos de uma maneira prática e efetiva os códigos de grupo devem ser constituídos de seqüências de simetrias, denominados códigos de sime-

\footnotetext{
*O autor está atualmente no Departamento de Telemática, sob licença do Departamento de Matemática, Universidade Nacional San Luis Gonzaga de Ica, Peru.

†O autor está no Departamento de Telemática, FEEC-UNICAMP, C.P. 6101, 13081-970, Campinas, SP, Brasil. Este trabalho foi financiado em parte pela Fundação de Amparo à Pesquisa do Estado de São Paulo - FAPESP, No. 95/4720-8, e em parte pelo Conselho Nacional de Desenvolvimento Científico e Tecnológico - CNPq, No. 301416/85-0, Brasil. palazzo@dt.fee.unicamp.br
}

trias. Um código de simetria $\mathcal{G}$ é um subgrupo do produto direto de um número infinito de grupos, isto é, $\oplus_{k \in \mathbb{Z}} G_{k}=$ $\ldots \ominus G_{-i \ominus} \ldots \ldots \oplus G_{-1 \oplus} G_{0 \ominus} \ldots G_{1 \ominus} \ldots \ldots \oplus G_{i \ominus} \ldots, i \epsilon$ $\mathbb{N}$, onde para cada $k \in \mathbb{Z}, G_{k} \subseteq \Gamma\left(S_{k}\right)$ é o grupo de simetrias do conjunto discreto $S_{k} \subset \mathbb{R}^{n_{k}}$.

O código Euclidiano $\mathcal{C}$ casado a $\mathcal{G} \subset \oplus_{k \in \mathbb{Z}} G_{k}$ é obtido a partir de uma sequiencia semente $\left\{x_{k}\right\}_{k \in \mathbb{Z}}, x_{k} \in \mathbb{R}^{n_{k}}$. Isto significa que cada palavra-código $\left\{y_{k}\right\}_{k \in \mathbb{Z}} \in \mathcal{C}$ é tal que para cada $k \in \mathbb{Z}, y_{k}=g_{k}\left(x_{k}\right), g_{k} \in G_{k}$. Estes códigos Euclidianos são denominados códigos geometricamente uniformes, [3].

Neste trabalho iremos considerar códigos invariantes no tempo e com um número finito de estados, isto é, um código tendo como base um conjunto discreto Euclidiano $S \subset \mathbb{R}^{n}$ com cardinalidade $|S|$ finita, e tal que $S_{k}=S \subset \mathbb{R}^{n}$ para todo $k \in \mathbb{Z}$. Neste caso, $G_{k}=G, \forall k \in \mathbb{Z}$ com $|G|$ finito. Agora, seja $G^{\mathbb{Z}}=\oplus_{k \in \mathbb{Z}} G_{k}, G_{k}=G, \forall k \in \mathbb{Z}$, considere um subgrupo $\mathcal{G} \subset G^{\mathbb{Z}}$. Assim, $\mathcal{G}$ é um código de simetrias invariante no tempo e com um número finito de estados. Esta classe de códigos de simetrias invariantes no tempo é o exemplo fundamental dos códigos de Schreier [11] e, em particular, dos códigos convolucionais abelianos.

Os códigos convolucionais abelianos são subgrupos de $G^{\mathbb{Z}}$, onde $G$ é um grupo finito arbitrário, não necessariamente um grupo de simetrias de algum conjunto Euclidiano discreto $S \subset \mathbb{R}^{n}$. Esta generalidade somente facilita a aplicação sobre grupos arbitrários.

Os códigos convolucionais abelianos admitem análise local, isto é, a análise da seção de treliça numa unidade do tempo é suficiente para determinar as propriedades tais como distância livre, controlabilidade, minimalidade, etc. de todo o código. Esta análise local permite determinar a relação equipotente entre a classe do produto direto e a classe dos códigos convolucionais abelianos, via os codificadores isomorfos. Isto significa que para cada código convolucional abeliano existe um único codificador isomorfo e para cada codificador isomorfo existe um único código convolucional abeliano.

Este trabalho está organizado da seguinte maneira. $\mathrm{Na} \mathrm{Se}-$ ção 2, apresentamos uma rápida descrição de máquinas no sentido da teoria dos autômatas, pois todos os codificadores considerados neste trabalho usam este modelo. Na Seção 3, motivados pela descrição matricial dos codificadores convolucionais binários, definimos os codificadores convolucionais elementares sobre grupos (CCE). A classe dos codificadores convolucionais lineares binários está contida na classe dos CCEs. Estudamos algumas propriedades dos CCEs que servem como guia para definir os codificadores convolucionais abelianos. É também proposto um teste simples de exclusão de CCEs catastróficos. Ainda nesta seção, apresentamos uma 


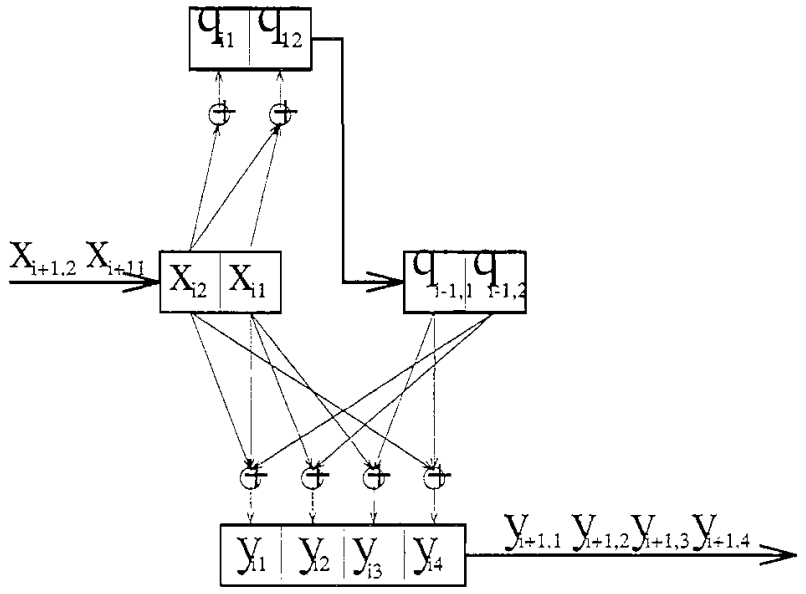

Figura 1. Máquina generalizada

proposta de redução de estados de um CCE, especialmente se este for catastrófico. Na Seção 4, estabelecemos as condições para a construção de codificadores convolucionais abelianos, onde propomos um algoritmo de construção e apresentamos um exemplo. Finalmente, na Seção 5, apresentamos as conclusões.

\section{MÁQUINAS}

A descrição de um sistema sob a excitação de alguma classe de entrada produzindo uma outra classe de saída tal que a sua estrutura interna possa também ser modificada, tem sido e será útil no modelamento de muitos dispositivos abstratos e fenômenos. Dentre as possíveis descrições, escolhemos aquela relativa à teoria dos autômatas, devido ao uso do conceito de máquinas, [5] e [9].

Definição 1 Uma máquina é uma quíntupla $M=$ $(X, Y, Q, \delta, \beta)$, onde

- X é o conjunto finito das entradas;

- Y é o conjunto finito das saídas;

- Q é o conjunto dos estados (não necessariamente finito);

- $\delta: X \times Q \rightarrow Q$ é o mapeamento do próximo estado;

- $\beta: X \times Q \rightarrow Y$ é o mapeamamento das saidas.

Esta definição é bastante geral no sentido de que máquinas podem caracterizar sistemas abstratos como por exemplo os sistemas de equações diferenciais, chegando até o processo evolutivo do comportamento de um ser humano. Willems, [4], usa de maneira precisa o termo comportamento de um sistema como sendo uma subclasse de sequências bi-infinitas de algum produto cartesiano $\prod_{k \in \mathbb{Z}} S_{k}$, onde cada $S_{k}$ é um corpo algébrico sendo este o alfabeto de saída da respectiva máquina $M_{k}$. A proposta de Trott [1], [10] sobre o conceito de comportamento de um sistema é no sentido de que cada $S_{k}$ seja um grupo algébrico. A noção de uma máquina generalizada, veja Figura 1., tendo como ponto de partida a Definição 1 , ocorre quando cada $S_{k}$ é um conjunto arbitrário podendo apresentar ou não alguma estrutura algébrica.

Seja $\Psi: X \times Q \rightarrow Q \times Y \times Q$ a aplicação definida por

$$
\Psi(x, q)=(q, \beta(x, q), \delta(x, q)) .
$$

Então, o conjunto $T \subset Q \times Y \times Q$, definido por

$$
T=\operatorname{Im}(\Psi)=\Psi(X \times Q),
$$

é chamado seção de treliça associada à máquina $M$, ou simplesmente a treliça de $M$. Cada elemento $t=$ $(q, \beta(x, q), \delta(x, q)) \in T$ é chamado uma transição ou um ramo da treliça.

Considere a classe de seqüências finitas do conjunto das entradas $X^{*}=\left\{x^{*}=\left\{x_{i}\right\}_{i=1}^{n}: x_{i} \in X n \in \mathbb{N}\right\}$. Dada uma seqüência finita $x^{*}=\left\{x_{i}\right\}_{i=1}^{n}$, denotamos o seu comprimento por $\left|x^{*}\right|$, e assim $\left|x^{*}\right|=n$.

Definição 2 Dizemos que a máquina $M=(X, Y, Q, \delta, \beta)$ é controlável se para todos $q$ e $q^{\prime} \in Q$, existir uma seqüencia finita $x^{*}$, com $1 \leq\left|x^{*}\right| \leq n$, tal que $q^{\prime}=\delta^{*}\left(x^{*}, q\right)$, onde

$$
\delta^{*}\left(x^{*}, q\right)=\delta\left(x_{n}, \delta\left(x_{n-1}, \ldots, \delta\left(x_{1}, q\right) \ldots\right)\right.
$$

Dado $j \in \mathbb{N}$, se para todos $q, q^{\prime} \in Q$ existir um $x^{*} \in X^{*}$ com $1 \leq\left|x^{*}\right| \leq j$ tal que $q^{\prime}=\delta^{*}\left(x^{*}, q\right)$ então, dizemos que a máquina é $j$-controlável. Dessa forma, fica fácil de precisar que se a máquina é $j$-controlável então, ela também será $(j+$ 1)-controlável.

O número $\nu=\min \{j: M$ é $j$-controlável $\}$ é o índice de controlabilidade de $M$. Na verdade, controlabilidade é uma propriedade da classe de sequiências de saída $\mathcal{C}$ da máquina $M$ ou, equivalentemente, do comportamento do sistema. Mas como para uma máquina $M$ existe uma única classe $\mathcal{C}$ de sequências de saída associada a $M$, então é natural dizer que $M$ é controlável para dizer que $\mathcal{C}$ é controlável. Assim, esta característica ocorrerá de maneira similar com as demais propriedades de $\mathcal{C}$. Isto é, quando $M$ apresentar uma certa propriedade $\mathcal{P}$, então $\mathcal{C}$ apresentará a mesma propriedade $\mathcal{P}$.

\section{CODIFICADORES CONVOLUCIONAIS ELEMENTARES SOBRE GRUPOS}

Sejam $k$ e $n$ números naturais. Seja $L$ uma matriz $k \times n$ definida por $L=\left(l_{i j}\right)$,onde $l_{i j} \in Z, 1 \leq i \leq k$ e $1 \leq$ $j \leq n$. Seja $G$ um grupo abeliano. Para qualquer $n \in N$, considere $G^{n}$ como sendo $n$ cópias de $G$, com a $G$-operação sobre as respectivas coordenadas. Então, $G^{n}$ é também um grupo abeliano. Dados $x \in G^{k}$ e $L$, defina o produto $x . L$ como sendo

$$
x . L=\left(\sum_{i=1}^{k} x_{i} l_{i 1}, \sum_{i=1}^{k} x_{i} l_{i 2}, \cdots, \sum_{i=1}^{k} x_{i} l_{i n}\right),
$$

onde

$$
x_{i} l_{i j} \triangleq \begin{cases}\overbrace{x_{i} * x_{i} * \ldots * x_{i}}^{l_{i j} \text {-termos }}, & \text { se } l_{i j}>0 \\ e_{G}, l_{i j} \text { termos } & \text { se } l_{i j}=0 \\ \overbrace{\left(x_{i} * x_{i} * \ldots * x_{i}\right)^{-1}}, & \text { se } l_{i j}<0 .\end{cases}
$$


Devido a condição abeliana de $G$ e $G^{n}$ podemos usar o símbolo de adição + em lugar do símbolo *, e também para o elemento identidade usar o símbolo $0 \mathrm{em}$ lugar de $e_{G}$. Sob estas condições temos a seguinte definição para o codificador convolucional elementar.

Definição 3 Sejam $n, k$ e $m$ números naturais tais que $n>$ $k \geq 1$ e $m \geq 1$. Considere as matrizes $L_{0}, L_{1}, \ldots . L_{m}$, com $L_{i}=\left(l_{r s}^{i}\right)$, onde $l_{r s}^{i} \in \mathbb{Z}, 1 \leq r \leq k, 1 \leq s \leq n$ $e i=0,1, \ldots, m$. Um codificador convolucional elementar (CCE) com parâmetros $(n, k, m)$ sobre $G$, onde $n$ é o comprimento da palavra-código de transição, $k$ é o número de dígitos de informação e $m$ a memória total, é uma máquina $M \triangleq(X, Y, Q, \delta, \beta)$ onde

- X $\subset G^{k}$ é o alfabeto das entradas;

- $Y \subset G^{n}$ é o alfabeto das saídas;

$-Q=\left\{q=\left(x_{1}, x_{2}, \ldots, x_{m}\right) ; x_{i} \in X\right\} \subset\left(G^{k}\right)^{m} \cong$ $G^{k m}$,é o conjunto (ou espaço) dos estados da máquina;

- $\delta: X \times Q \rightarrow Q$, é uma aplicação definida por $\delta\left(x_{0} ; q\right)=\delta\left(x_{0} ; x_{1}, x_{2}, \ldots, x_{m-1}, x_{m}\right)=$ $\left(x_{0} ; x_{1}, x_{2}, \ldots, x_{m-1}\right)$ (aplicação do próximo estado);

- $\beta: X \times Q \rightarrow Y$, é uma aplicação definida por $\beta\left(x_{0} ; q\right)=\beta\left(x_{0} ; x_{1}, \ldots, x_{m}\right)=x_{0} L_{0}+x_{1} L_{1}+\ldots+$ $x_{m} L_{m}$ (aplicação das saídas).

Note que um CCE é um exemplo de máquina, isto é, satisfaz a Definição 1. Desse modo, a classe dos CCEs está contida na classe das máquinas.

Da Definição 3, podemos extrair as seguintes propriedades dos codificadores convolucionais elementares.

Proposição 4 Se $X$ é um grupo, então

i) $Q$ e $\beta\left(X_{\Theta} Q\right) \subset Y$ são grupos.

ii) O produto cartesiano $X \times Q$ converte-se em um produto direto de grupos e as funções $\delta$ e $\beta$ são homomorfismos de grupos, com $\delta$ sendo sobrejetora.

iii) Os conjuntos $Y_{0}=\left\{\beta\left(x, e_{Q}\right)\right\}_{x \in X}$ e $Y_{1}=$ $\left\{\beta(x, q): \delta(x, q)=e_{Q}\right\}$ são subgrupos normais de $\beta(X, Q)$. Além disso, $\frac{\beta(X, Q)}{Y_{0}} \cong \frac{\beta(X, Q)}{Y_{1}} \cong Q$.

iv) O CCE é uma máquina controlável, com indice de controlabilidade $\nu \leq m$.

Prova :

i) Dados $y=\sum_{i=0}^{m} x_{i} L_{i} \in Y$ e $y^{\prime}=\sum_{i=0}^{m} x_{i}^{\prime} L_{i} \in Y$, temos que $y+y^{\prime}=\sum_{i=0}^{m}\left(x_{i} L_{i}+x_{i}^{\prime} L_{i}\right)=\sum_{i=0}^{m} x_{i}^{\prime \prime} L_{i} \in$ $Y$, pois $X$ é um grupo. Analogamente, dados $q=$ $\left(x_{1}, x_{2}, \ldots, x_{m}\right)$ e $q^{\prime}=\left(x_{1}^{\prime}, x_{2}^{\prime}, \ldots, x_{m}^{\prime}\right)$, temos que $q+q^{\prime}=$ $\left(x_{1}+x_{1}^{\prime}, x_{2}+x_{2}^{\prime}, \ldots, x_{m}+x_{m}^{\prime}\right) \in Q$, pois Xé um grupo.

ii) Como $X$ e $Q$ são grupos, o produto direto $X_{\ominus} Q$ é um grupo. Assim, $\delta$ é um mapeamento entre dois grupos. Sejam $(x, q)$ e $\left(x^{\prime}, q^{\prime}\right)$ dois elementos de $X \times Q$, com $q=\left(x_{1}, x_{2}, \ldots, x_{m}\right) e q^{\prime}=\left(x_{1}^{\prime}, x_{2}^{\prime}, \ldots, x_{m}^{\prime}\right)$. Então,

$$
\begin{aligned}
& \delta\left((x, q)+\left(x^{\prime}, q^{\prime}\right)\right)=\delta\left(x+x^{\prime}, q+q^{\prime}\right) \\
& =\left(x+x^{\prime}, x_{1}+x_{1}^{\prime}, x_{2}+x_{2}^{\prime}, \ldots, x_{m}+x_{m}^{\prime}\right) \\
& =\left(x, x_{1}, x_{2}, \ldots, x_{m}\right)+\left(x^{\prime}, x_{1}^{\prime}, x_{2}^{\prime}, \ldots, x_{m}^{\prime}\right) \\
& =\delta(x, q)+\delta\left(x^{\prime}, q^{\prime}\right) .
\end{aligned}
$$

Portanto, $\delta$ é um homomorfismo de grupos. Por outro lado, dado $q=\left(x_{1}, x_{2}, \ldots, x_{m}\right) \in Q$, assuma $q_{0}=$ $\left(x_{2}, x_{3}, \ldots, x_{m+1}\right) \in Q$ e $x_{1} \in X$. Então, $\delta\left(x_{1}, q_{0}\right)=q$. Com isso, temos que $\delta$ é sobrejetora.

De maneira análoga, podemos mostrar que $\beta$ é também um homomorfismo de grupos.

iii) Defina o mapeamento auxiliar $\psi: \beta\left(X_{\ominus} Q\right) \rightarrow Q$ por $\psi(\beta(x, q)) \triangleq q$. Então,

$$
\begin{aligned}
& \psi\left(\beta(x, q)+\beta\left(x^{\prime}, q^{\prime}\right)\right)=\psi\left(\beta\left(x+x^{\prime}, q+q^{\prime}\right)\right) \\
& =q+q^{\prime}=\psi(\beta(x, q))+\psi\left(\beta\left(x^{\prime}, q^{\prime}\right)\right) .
\end{aligned}
$$

Assim, ¿́ ú um homomorfismo sobrejetor. Consequentemente, temos que

$$
\operatorname{Ker}(\psi)=\left\{\beta(x, q): q=\psi(\beta(x, q))=\epsilon_{Q}\right\}=Y_{0}
$$

Logo, pelo teorema fundamental dos homomorfismos, [6] $e[7]$, concluimos que $\frac{B\left(X_{\Phi} Q\right)}{Y_{0}} \cong Q$.

A prova para $Y_{1}$ é análoga. Neste caso definimos o mapeamento auxiliar $\psi: \beta\left(X_{\ominus} Q\right) \rightarrow Q$ como $\psi(\beta(x, q)) \triangleq$ $\delta(x, q)$

iv) Dados os estados $q=\left(x_{1}, x_{2}, \ldots, x_{m}\right)$ e $q^{\prime}=$ $\left(x_{1}^{\prime}, x_{2}^{\prime}, \ldots, x_{m}^{\prime}\right)$, considere a seqüência finita $x^{*}=$ $x_{1}^{\prime} x_{2}^{\prime} \ldots x_{m}^{\prime} \in X^{*}$, temos que,

$$
q^{\prime}=\delta^{*}\left(x^{*}, q\right)
$$

Portanto, $M$ é sempre $m$-controlável.

A seguir, iremos consdiderar $X$ como sendo um grupo. Portanto, todas as propriedades da Proposição 4 continuam válidas.

Lema 5 A seção de treliça do CCE, não possui transições paralelas. Portanto, o mapeamento $\Psi$, dado em (1), é injetor.

Prova : Lembremos que duas transições $t_{1}=$ $\left(q_{1}, \beta\left(x_{1}, q_{1}\right), \delta\left(x_{1}, q_{1}\right)\right) \in T e t_{2}=\left(q_{2}, \beta\left(x_{2}, q_{2}\right)\right.$, $\left.\delta\left(x_{2}, q_{2}\right)\right) \in T$ são ditas transições paralelas se $q_{1}=q_{2}$ e $\delta\left(x_{1}, q_{1}\right)=\delta\left(x_{2}, q_{2}\right)$. Agora, se a treliça do CCE tiver $t_{1}$ e $t_{2}$ como transições paralelas, então existe um $q \in Q$ tal que $t_{1}=\left(q, \beta\left(x_{1}, q\right), \delta\left(x_{1}, q\right)\right) e$ $t_{2}=\left(q, \beta\left(x_{2}, q\right), \delta\left(x_{2}, q\right)\right) \operatorname{com} \delta\left(x_{1}, q\right)=\delta\left(x_{2}, q\right)$. Disto, temos que $\delta\left(x_{1}-x_{2}, 0\right)=0 \in G^{k m}$. Isto implica pela definiçâo de $\delta$, que $x_{1}=x_{2}$. Logo, $t_{1}=t_{2}$.

Seja $M=(X, Y, Q, \delta, \beta)$. Considere uma seqüiência de entradas $\left\{x_{i}\right\}_{i=1}^{\infty}, x_{i} \in X$, e $q_{0}$ o estado inicial, com $q_{0} \in$ $Q$. Seja $\left\{q_{i}\right\}_{i=1}^{\infty}, q_{i} \in Q$, a sequiência de estados gerada por $\left\{x_{i}\right\}_{i=1}^{\infty}$ através de $M$, definida por

$$
\begin{aligned}
& q_{1}=\delta\left(x_{1}, q_{0}\right) \\
& q_{2}=\delta\left(x_{2}, q_{1}\right) \\
& \vdots \\
& q_{i}=\delta\left(x_{i}, q_{i-1}\right)
\end{aligned}
$$

Seja $\left\{y_{i}\right\}_{i=1}^{\infty}, y_{i} \in Y$ a sequiência da saida gerada por $\left\{x_{i}\right\}_{i=1}^{\infty}$ através de $M$, definida por 


$$
\begin{aligned}
& y_{1}=\beta\left(x_{1}, q_{0}\right) \\
& y_{2}=\beta\left(x_{2}, q_{1}\right) \\
& \vdots \\
& y_{i}=\beta\left(x_{i}, q_{i-1}\right)
\end{aligned}
$$

Definição 6 Dado o $C C E=(X, Y, Q, \delta, \beta)$, o código convolucional $C$ associado ao CCE é a família de sequiências $\left\{y_{i}\right\}_{i=1}^{\infty}$ definida em (3.).

Cada sequiência $\left\{y_{i}\right\}_{i=1}^{\infty}$ é chamada de palavra-código. Este código é invariante no tempo, pois é produzido por um único $\mathrm{CCE}$

Definição 7 Seja $\mathcal{C}$ um código qualquer. Seja $\left\{C_{i}\right\}_{i \in \mathbb{N}} a$ familia de codificadores associados com o código C. Seja $\left\{s_{i}\right\}_{i \in \mathbb{N}}$ a familia de números naturais tal que, cada $s_{i}$ é a cardinalidade dos estados de cada codificador $C_{i}$. Então, um codificador $C_{j}$ é dito mínimo quando seu número de estados $s_{j}$ é minimo, isto é, $s_{j} \leq s_{i}$, para todo $i \in \mathbb{N}$.

Quando da determinação de codificadores dos códigos de treliça, é importante que sejam estabelecidas as condições de eliminação de códigos catastróficos, pois os mesmos, são tais que para um número finito de erros introduzidos pelo canal conduzem a um número infinito de erros na decodificação. O Teorema 3.4 de [2], válido para códigos completos, veio resolver este problema. A versão deste teorema para os CCEs é a seguinte,

Teorema 8 Seja a máquina $M=(X, Y, Q, \delta, \beta)$ tal que $X=G^{k}, Y=G^{n}$ e $Q=G^{k m}$, onde $G$ é um grupo finito com carateristica $p$ tal que o $\operatorname{mdc}(p,(m+1))=1$. Sejam $L_{0}, \ldots, L_{m}$ as matrizes que definem $\beta$. Então, o código $\mathcal{C}$ associado com $M$ é não catastrófico se, e somente se, $L=\sum_{i=0}^{m} L_{i}$ é tal que $x . L \neq 0 \in G^{n}$ e $m+1$ não é um múltiplo da caraterística do grupo $G$, para todo $x \in X$ tal que $x \neq 0$.

Prova : Seja $t \in T$ uma transição horizontal definida como sendo $t=(q, \beta(x, q), \delta(x, q))$, tal que $\delta(x, q)=q$. Se $q=\left(x_{1}, \ldots, x_{m}\right)$, temos que $\delta(x, q)=q$ se, e somente se, $x=x_{1}=\cdots=x_{m}$.

Em [2] é provado que para o codificador ser mínimo uma condição necessária e suficiente é que a única transição horizontal rotulada com $0 \in Y$ deve ser a transição trivial $(0, \beta(0,0), \delta(0,0))$. Portanto, se $(x, q) \in X_{\oplus Q}$ é tal que $q=\delta(x, q)$ teremos que $\beta(x, q)=x L_{0}+x L_{1}+\ldots x L_{m}=(m+1) x L_{\text {. }}$.

Dessa forma, o Teorema 8 é um critério de se evitar catastroficidade na construção do CCE. Por outro lado, o código catastrófico poderá ser transformado em um código não catastrófico através da determinação do correspondente codificador mínimo. Para isso necessitamos de técnicas que permitam a redução da cardinalidade dos estados.

\subsection{REDUÇĀO DOS ESTADOS}

Nesta subseção assumiremos que o mapeamento $\beta$ é sobrejetor, isto é, $\beta\left(X_{\Theta} Q\right)=Y$.
Proposição 9 Seja $Q^{\prime} \subset Q$ um subgrupo normal de Q. Se $Y^{\prime}$ é definido como $Y^{\prime}=$ $\left\{\beta(x, q) \in Y: x \in X\right.$ eq $\left.q, \delta(x, q) \in Q^{\prime}\right\}$, então $Y^{\prime} \quad e ́$ um subgrupo normal de $Y$.

Prova : Defina a aplicação auxiliar $\psi: Y \rightarrow \frac{Q}{Q^{\prime}} \times \frac{Q}{Q^{\prime}}$ como sendo

$$
\psi(\beta(x, q)) \triangleq\left(q+Q^{\prime}, \delta(x, q)+Q^{\prime}\right) .
$$

Então,

$$
\begin{aligned}
& \psi\left(\beta(x, q)+\beta\left(x^{\prime}, q^{\prime}\right)\right)= \\
& =\psi\left(\beta\left(x+x^{\prime}, q+q^{\prime}\right)\right) \\
& =\left(\left(q+q^{\prime}\right)+Q^{\prime}, \delta\left(x+x^{\prime}, q+q^{\prime}\right)+Q^{\prime}\right) \\
& =\left(\left(q+Q^{\prime}\right), \delta(x, q)+Q^{\prime}\right)+\left(\left(q^{\prime}+Q^{\prime}\right), \delta\left(x^{\prime}, q^{\prime}\right)+Q^{\prime}\right) \\
& =\psi(\beta(x, q))+\psi\left(\beta\left(x^{\prime}, q^{\prime}\right)\right) .
\end{aligned}
$$

Por outro lado,

$$
\begin{aligned}
\operatorname{Ker}(\psi) & =\left\{\beta(x, q): \psi(\beta(x, q))=\left(Q^{\prime}, Q^{\prime}\right)\right\} \\
& =\left\{\beta(x, q) \in Y: q \in Q^{\prime} \text { e } \delta(x, q) \in Q^{\prime}\right\}=Y^{\prime} .
\end{aligned}
$$

Assim, $Y^{\prime}$ é normal em $Y$.

Definição 10 Dada uma máquina $M=(X, Y, Q, \delta, \beta)$, sejam $Y^{\prime} \subset Y$ e $Q^{\prime} \subset Q$, definidos como na Proposição $9, e$ tal que $\delta(0, q) \in Q^{\prime}$, para todo $q \in Q^{\prime}$. Então, definimos a máquina $M^{\prime}=\left(X, \frac{\beta\left(X_{Q} Q\right)}{Y^{\prime}}, \frac{Q}{Q^{\prime}}, \delta^{\prime}, \beta^{\prime}\right)$ onde

- $\delta^{\prime}: X \times \frac{Q}{Q^{\prime}} \rightarrow \frac{Q}{Q^{\prime}}$ é dado por $\delta^{\prime}\left(x, q+Q^{\prime}\right)=\delta(x, q)+$ $Q^{\prime}$ :

- $\beta^{\prime}: X \times \frac{Q}{Q^{\prime}} \rightarrow \frac{Y}{Y^{\prime}}$ é dado por $\beta^{\prime}\left(x, q+Y^{\prime}\right)=\beta(x, q)+$ $Y^{\prime}$ (classe das transições paralelas).

Geralmente, uma máquina $M^{\prime}$ não é um CCE, pois se $Y^{\prime}$ é um subgrupo não trivial, então $M^{\prime}$ possui transições paralelas. O Exemplo 14, a seguir, ilustra esta afirmação.

Proposição 11 As aplicações $\delta^{\prime}$ e $\beta^{\prime}$ têm as seguintes propriedades:

- $\delta^{\prime}$ e $\beta^{\prime}$ são bem definidas, i.e., elas não dependem da escolha do representante da classe $q+Q^{\prime}$.

- $\delta^{\prime}$ e $\beta^{\prime}$ são homomorfismos de grupos com $\delta^{\prime}$ sendo sobrejetora.

- $\beta^{\prime}$ é tal que os conjuntos

$$
Y_{Q_{0}^{\prime}}=\left\{\beta^{\prime}\left(x, Q^{\prime}\right): x \in X\right\},
$$

e

$$
\begin{gathered}
Y_{Q_{1}^{\prime}}=\left\{\beta^{\prime}\left(x, q+Q^{\prime}\right):\left(x, q+Q^{\prime}\right) \in X \times \frac{Q}{Q^{\prime}},\right. \\
\left.\delta^{\prime}\left(x, q+Q^{\prime}\right)=Q^{\prime}\right\},
\end{gathered}
$$

são subgrupos normais de $\frac{Y}{Y^{\prime}}$. Além disso,

$$
\frac{\frac{Y}{Y^{\prime}}}{Y_{Q_{0}^{\prime}}} \cong \frac{\frac{Y}{Y^{\prime}}}{Y_{Q_{1}^{\prime}}} \cong \frac{Q}{Q^{\prime}}
$$


Prova :

- Se $q, q_{1} \in q+Q^{\prime}$, então $\delta^{\prime}\left(x, q+Q^{\prime}\right)=\delta(x, q)+Q^{\prime} e$ $\delta^{\prime}\left(x, q_{1}+Q^{\prime}\right)=\delta\left(x, q_{1}\right)+Q^{\prime}$. Logo, $\delta^{\prime}\left(x, q+Q^{\prime}\right)-$ $\delta^{\prime}\left(x, q_{1}+Q^{\prime}\right)=\delta\left(0, q-q_{1}\right)+Q^{\prime}$. Como $\delta(0, q) \in Q^{\prime}$ para todo $q \in Q^{\prime}$, temos que $\delta^{\prime}\left(x, q+Q^{\prime}\right)-\delta^{\prime}\left(x, q_{1}+Q^{\prime}\right)=$ $Q^{\prime}$.

A prova para $\beta^{\prime}$ é similar, pois pela definição de $Y^{\prime}$, $\beta(0, q) \in Y^{\prime}$ para todo $q \in Q^{\prime}$.

$-\delta^{\prime}\left(x, q+Q^{\prime}\right)+\delta^{\prime}\left(x_{1}, q_{1}+Q^{\prime}\right)=\delta(x, q) \div \delta\left(x_{1}, q_{1}\right)+Q^{\prime}=$ $\delta\left(x+x_{1}, q+q_{1}\right)+Q^{\prime}=\delta^{\prime}\left(x+x_{1}, q+q_{1}+Q^{\prime}\right)$. A prova para $\beta^{\prime}$ é similar.

\subsection{EXEMPLOS}

Nesta seção, iremos apresentar alguns exemplos de modo a ilustrar os conceitos estabelecidos.

Exemplo 12 Dados $G=\mathbb{Z}_{2}, n=3, k=2, \quad m=2$, e $L_{0}=\left(\begin{array}{ccc}1 & 0 & 1 \\ 1 & 1 & 0\end{array}\right) ; \quad L_{1}=\left(\begin{array}{ccc}0 & 1 & 0 \\ 0 & 1 & 1\end{array}\right) ; L_{2}=$ $\left(\begin{array}{lll}1 & 0 & 1 \\ 0 & 1 & 1\end{array}\right)$. Considere a máquina $M$ dada por

$$
\begin{aligned}
& X=\mathbb{Z}_{2}^{2}=\{00,01,10,11\} \\
& Q=X^{2}=\mathbb{Z}_{2}^{4}=\left\{\begin{array}{lllll}
0000 & 0100 & 1000 & 1100 & 0001 \\
0010 & 0110 & 1010 & 1110 & 0011 \\
0101 & 1001 & 1101 \\
0111 & 1011 & 1111
\end{array}\right\} \\
& Y=\mathbb{Z}_{2}^{3}=\{000,001,010,100,011,110,101,111\} \\
& \delta\left(x_{0}, q\right)=\delta\left(x_{0},\left(x_{1}, x_{2}\right)\right)=\left(x_{0}, x_{1}\right), \text { com } x_{i} \in \mathbb{Z}_{2}^{2} \\
& \beta\left(x_{0}, q\right)=\beta\left(x_{0},\left(x_{1}, x_{2}\right)\right)=x_{0} L_{0}+x_{1} L_{1}+x_{2} L_{2}
\end{aligned}
$$

A seção de treliça da máquina $M=\left(\mathbb{Z}_{2}^{2}, \mathbb{Z}_{2}^{3}, \mathbb{Z}_{2}^{4}, \delta, \beta\right)$ é mostrada na Figura 2.. O código associado é um código convolucional binário com distância $d_{f r e e}=3$, taxa $R_{C}=2 / 3$ e memória 2 , onde $d_{\text {free }}$ é a menor distância de Hamming entre todas as palavras do código.

Exemplo 13 Considere os grupos $X=\mathbb{Z}_{2}^{2}, Y=\mathbb{Z}_{2}^{3}$, $Q=\mathbb{Z}_{2}^{2}$ e as matrizes $L_{0}=\left(\begin{array}{lll}1 & 1 & 0 \\ 0 & 1 & 1\end{array}\right)$ e $L_{1}=$ $\left(\begin{array}{lll}1 & 1 & 0 \\ 1 & 0 & 0\end{array}\right)$. O mapeamento $\delta: X_{\oplus} Q \rightarrow Q$ é dado por $\delta(x, q)=x$. O mapeamento $\beta: X_{\oplus} Q \rightarrow Y$ é dado por $\beta(x, q)=x L_{0}+q L_{1}$. A seção de treliça desta máquina $M=\left(\mathbb{Z}_{2}^{2}, \mathbb{Z}_{2}^{3}, \mathbb{Z}_{2}^{2}, \delta, \beta\right)$ é mostrado na Figura 3. . O código associado possui $d_{\text {free }}=3, R_{C}=\frac{2}{3}$ e memória 2 . Este código é catastrófico, pois para o estado inicial 01 a seqüência ....0101010101 é codificada na palavra código $00000000 \ldots .$.

Exemplo 14 Dado o CCE do Exemplo 13, considere o subgrupo normal $Q^{\prime}$ de $Q$, denotado por $Q^{\prime}<Q$, e especificado por $Q^{\prime}=\{00,10\}$. Então $Y^{\prime}<Y$ é dado por $Y^{\prime}=\{000,110\}$. Com isso, as classes determinadas por $Q^{\prime}$ são

$$
\begin{array}{ll}
Q^{\prime} & =\{00,10\} \\
01+Q^{\prime} & =\{01,11\} .
\end{array}
$$

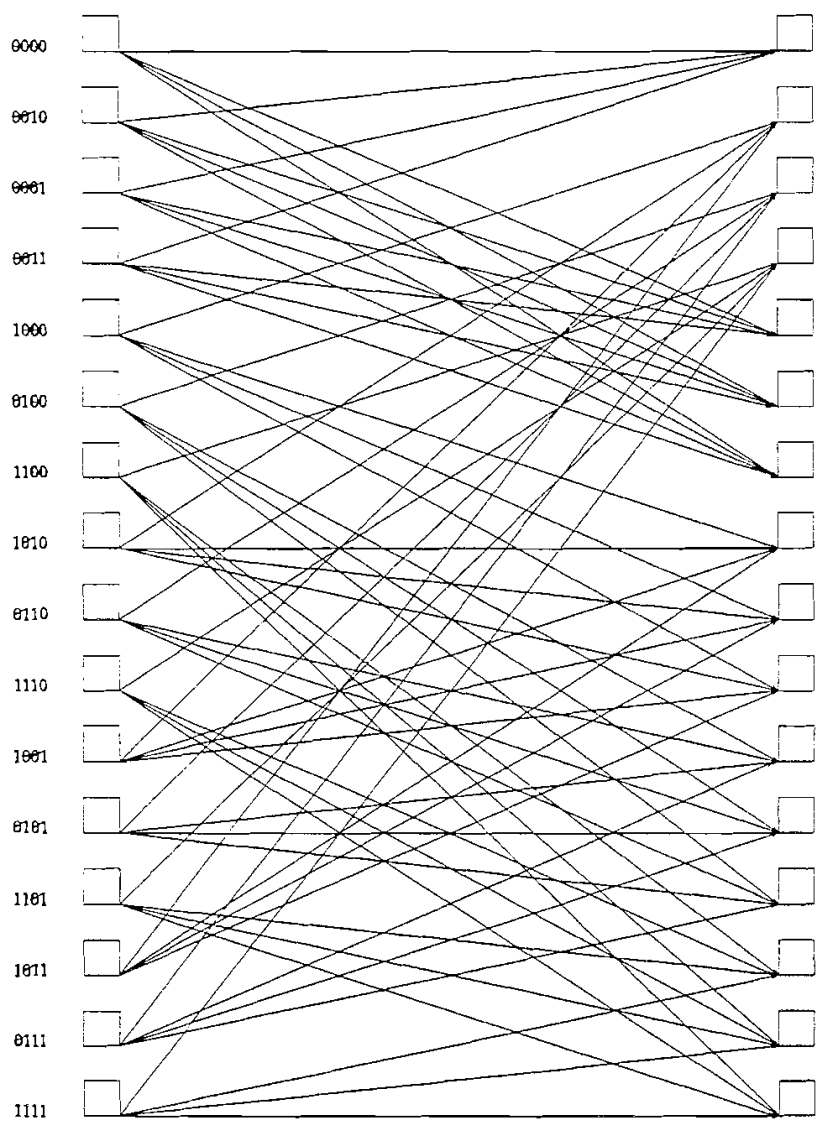

Figura 2. Treliça para o CCE do Exemplo 12

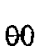

$\theta 1$

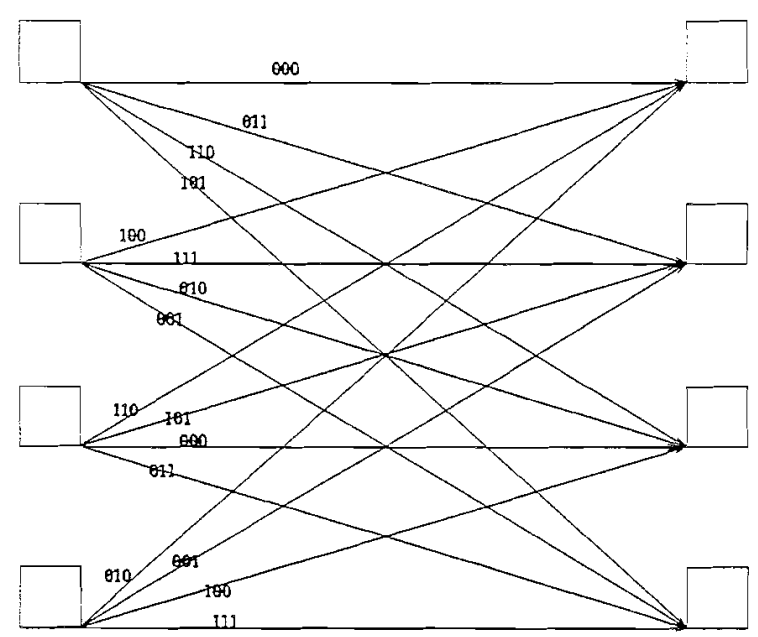

Figura 3. Treliça para o CCE do Exemplo 13 


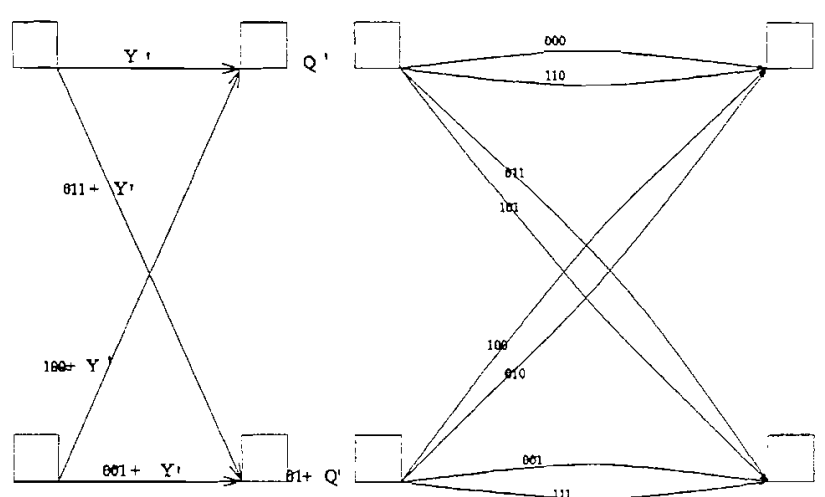

Figura 4. Treliça para a máquina do Exemplo 14.

As classes determinadas por $Y^{\prime}$ são:

$$
\begin{array}{ll}
Y^{\prime} & =\{000,110\} \\
100 \div Y^{\prime} & =\{100,010\} \\
001+Y^{\prime} & =\{001,111\} \\
011+Y^{\prime} & =\{011,101\}
\end{array}
$$

Então a nova máquina $\left(X, \frac{Y}{Y^{\prime}}, \frac{Q}{Q^{\prime}}, \delta^{\prime}, \beta^{\prime}\right)$ é especificada pelo mapeamento $\delta^{\prime}: X_{\oplus} \frac{Q}{Q^{\prime}} \rightarrow \frac{Q}{Q^{\prime}}$ definido por $\delta^{\prime}\left(x, q+Q^{\prime}\right)=$ $\delta(x, q)+Q^{\prime}$ e pelo mapeamento $\beta^{\prime}: X_{\ominus} \frac{Q}{Q^{\prime}} \rightarrow \frac{Y}{Y^{\prime}}$ definido por $\beta^{\prime}\left(x, q+Q^{\prime}\right)=\beta(x, q)+Y^{\prime}$, onde $\delta$ e $\beta$ estão definidos no Exemplo 13.

Uma representação da seção de treliça desta máquina é mostrada no lado esquerdo da Figura 4.

No lado direito da mesma figura, é mostrado a representação completa, isto é, os elementos das classes de $Y^{\prime \prime}$. Nenhuma destas treliças corresponde à de um CCE. Considerando que $\left\{Q^{\prime}, 01+Q^{\prime}\right\} \cong \mathbb{Z}_{2}$, a treliça do lado direito é produzida pela máquina $M=\left(\mathbb{Z}_{2}^{2}, \mathbb{Z}_{2}^{3}, \mathbb{Z}_{2}, \delta, \beta\right)$ onde $\delta: \mathbb{Z}_{2}^{2} \oplus \mathbb{Z}_{2} \rightarrow \mathbb{Z}_{2}$ é definido por $\delta\left(x_{1}, x_{2} ; q\right)=x_{2}, e \beta: \mathbb{Z}_{2}^{2} \oplus$ $\mathbb{Z}_{2} \rightarrow \mathbb{Z}_{2}^{3}$ é definido por $\beta\left(x_{1}, x_{2} ; q\right)=\left(x_{1}+q, x_{1}+x_{2}, x_{2}\right)$. Esta máquina produz o mesmo código que o CCE do Exemplo 13. Note que esta máquina näo satisfaz todas as propriedades do CCE (por exemplo a cardinalidade dos estados é menor do que a cardinalidade do grupo de entradas). Por isso uma definição mais geral é necessária.

Definição 15 Um codificador abeliano é uma máquina $M_{\oplus}=(X, Y, Q, \delta, \beta)$, onde $X, Y, Q$ são grupos abelianos finitos, $e \delta: X_{\oplus} Q \rightarrow Q$ e $\beta: X_{\oplus} Q \rightarrow Y$ são tais que

- Sé homomorfismo sobrejetor;

- $\beta$ é um homomorfismo tal que a aplicação $\Psi$, dada em (1), é injetora.

Assim, o CCE é um caso particular do codificador abeliano. Porém, um codificador abeliano não está definido para grupos não abelianos. Na próxima seção iremos estabelecer as condições para a construção de codificadores abelianos.

\section{CONSTRUÇÃO DE CODIFICADORES CONVOLUCIONAIS ABELIANOS}

Seja $H_{\oplus} K$ o produto direto de $H$ e $K$. Seja $\Phi_{K}$ a classe de homomorfismos sobrejetores entre $H_{\oplus} K$ e $K$, isto é,

$$
\Phi_{K}=\left\{\phi: H_{\oplus} K \rightarrow K: \phi \dot{e} \text { sobrejetora }\right\} .
$$

Seja $\mathcal{X}$ uma familia de subconjuntos de $H_{\ominus} K$, definido por

$$
\mathcal{X}=\left\{S: S=\mathbb{K} \operatorname{er}(\phi), \phi \in \Phi_{K}\right\} .
$$

Então, pelo teorema fundamental dos homomorfismos [6], [7], para cada $S \in \mathcal{X}$ temos que $S \triangleleft H_{\ominus} K$ e $\frac{H_{\oplus} K}{S} \cong K$.

Seja $H_{0}$ o subconjunto de $H_{\oplus} K$ definida por

$$
H_{0}=\left\{\left(h, e_{K}\right): h \in H\right\} \text {. }
$$

Então, $H_{0} \in \mathcal{X}$ pois $H_{0}=K \operatorname{Ker}\left(p_{2}\right)$, onde $p_{2}: H_{\oplus} K \rightarrow K$ é a projeção dada por $p_{2}(h, k)=k$. Consequentemente, $p_{2} \in$ $\Phi_{K}$ e $\mathcal{X}$ é uma subclasse não vazia. Veremos que para efeito de construção de bons códigos, $\mathcal{X}$ terá que ter mais do que un elemento.

Considere a classe $\mathcal{X}$ definida em (6). Defina $H_{1} \in \mathcal{X}$ como sendo

$$
H_{1}=\operatorname{Ker}(\delta) .
$$

Os subgrupos $H_{0}$ e $H_{1}$, onde $H_{0}$ é definido em (7), são fundamentais na construção de codificadores, como veremos a seguir.

Sejam $G$ e $H$ grupos tais que $H$ é um subgrupo normal de $G$, isto é, $H \triangleleft G$. Seja $\mathcal{N}_{G}$ a classe dos subgrupos normais de $G$, isto é,

$$
\mathcal{N}_{G}=\{H: H \varangle G\} .
$$

Omitiremos a demonstração do próximo teorema, pois a mesma é simples.

Teorema 16 Se $N \in \mathcal{N}_{G}$, então $G \cong N_{\oplus} \frac{G}{N}$

Lema 17 Seja $Q_{0 i} \subset Q$ definido por $Q_{0 i}=$ $\left\{q \in Q: \delta_{i}\left(\stackrel{(i)}{x}, e_{Q}\right)=q\right\}$, onde $\stackrel{(i)}{x}=\left(x_{j}, x_{j+1}\right.$,

$\left.\ldots, x_{j+1-i}\right)$. Então $Q_{0 i} \triangleleft Q$.

Prova : Seja $H_{i}^{\prime}=\left\{\left(\stackrel{(i)}{x}, e_{Q}\right): \stackrel{(i)}{x} \in X^{i}\right\}$, onde $X^{i}$ denota $i$ cópias de $X$, então considerando a projeção $p_{2}: X_{\ominus}{ }_{\ominus} Q \rightarrow Q$ dada por $p_{2}(\stackrel{(i)}{x}, q)=q$, temos que $p_{2}$ é um homomorfismo sobrejetor com Ker $\left(p_{2}\right)=H_{i}^{\prime}$. Logo, $H_{i}^{\prime} \triangleleft X_{\oplus}^{i} Q$. Por outro lado, uma vez que $\delta$ é sobrejetora temos que $\delta_{i}$ também é sobrejetora. Logo, $\delta_{i}\left(H_{i}^{\prime}\right) \triangleleft Q$ (a imagem de um subgrupo normal por um homomorfismo sobrejetor é também normal). Consequentemente, $\delta_{i}\left(H_{i}^{\prime}\right)=\left\{\delta_{i}\left(\stackrel{(i)}{x}, e_{Q}\right): \stackrel{(i)}{x} \in X^{i}\right\}=Q_{0 i}$

Lema 18 Dado $q \in \quad Q$, seja $Q_{q i}=$ $\left\{\delta_{i}(\stackrel{(i)}{x}, q): \stackrel{(i)}{x} \in X^{i}\right\}$. Então, $Q_{q i}$ é uma classe lateral de $Q_{0 i}$. Portanto, $\left|Q_{0 i}\right|=\left|Q_{q i}\right|$. 
Prova : Considere a classe $(\stackrel{(i)}{u}, q) \cdot H_{i}^{\prime}$ de $H_{i}^{\prime}$. Então, $\quad(\stackrel{(i)}{u}, q) \cdot H_{i}^{\prime}=(\stackrel{(i)}{u}, q) \cdot\left\{\left(\stackrel{(i)}{x}, \in_{Q}\right): \stackrel{(i)}{x} \in X^{i}\right\}$ $=\left\{(\stackrel{(i)}{x}, q): \stackrel{(i)}{x} \in X^{i}\right\} . \quad$ Logo, $\quad \delta_{i}\left((\stackrel{(i)}{u}, q) \cdot H_{i}\right)=$ $\left\{\delta_{i}(\stackrel{(i)}{x}, q): \stackrel{(i)}{x} \in X^{i}\right\}=Q_{q i}$. Por outro lado, $\delta_{i}\left((\stackrel{(i)}{u}, q) \cdot H_{i}^{\prime}\right)=\delta_{i}(\stackrel{(i)}{u}, q) \cdot \delta_{i}\left(H_{i}^{\prime}\right)=\delta_{i}(\stackrel{(i)}{u}, q) \cdot Q_{0 i}$. Assim, $Q_{q i}=\delta_{i}(\stackrel{(i)}{u}, q)$. $Q_{0 i}$. Isto mostra que $Q_{q i}$ é uma classe lateral de $Q_{0 i}$ e que, portanto, $\left|Q_{0 i}\right|=\left|Q_{q i}\right|$.

Teorema 19 Seja $Q_{0 i}<Q$ o subgrupo normal de $Q$ definido no Lema 17. Então, $M_{\oplus}=(X, Y, Q, \delta, \beta)$ é controlável se, e somente se, $Q_{0 i}=Q$, para algum $1 \leq i<|Q|$.

Prova : Suponha $M_{\ominus}$ controlável. Então, se

$$
\begin{aligned}
i= & \max \left\{j \in \mathbb{N}, j<|Q|: \delta_{j}\left(\stackrel{(j)}{x}, e_{Q}\right)=q,\right. \\
& \left.\stackrel{(j)}{x} \in X^{j}, q \in Q\right\}
\end{aligned}
$$

teremos ${ }^{1}$ que para todo $q \in Q$ existe $\stackrel{(i)}{x} \in X^{i}$ tal que $\delta_{i}\left(\stackrel{(i)}{x}, e_{Q}\right)=q$, pois se $q=\delta_{j}\left(\stackrel{(j)}{u}, e_{Q}\right)$ para $j<i$, então tomando $\stackrel{(i)}{x}=x_{i}, \ldots, x_{1+i-j}, \stackrel{(i-j)}{x}$, onde $\stackrel{(i-j)}{x}=$ \{elementoidentidade de $\left.X^{i-j}{ }_{\ominus} Q\right\}$, e para $s>i-j$, considere $x_{s}$ como sendo $x_{s}=u_{s-(i-j)}$. Temos então que $q=\delta_{i}\left(\stackrel{(i)}{x}, e_{Q}\right)$. Portanto, para todo $q \in Q$ temos que $q \in Q_{0 i}$.

Na outra direção do teorema, suponha que $Q=Q_{0 i}$, para algum $i \geq 1$. Então, dados $q$ e $r \in Q$, considere a classe $Q_{q i}=\left\{\delta_{i}(\stackrel{(i)}{x}, q): \stackrel{(i)}{x} \in X^{i}\right\}$ definida no Lema 18. Como $\left|Q_{0 i}\right|=\left|Q_{q i}\right|=|Q|$, então existe $\stackrel{(i)}{u} \in X^{i}$ tal que $\delta_{i}(\stackrel{(i)}{x}, q)=r$.

Teorema 20 (Construção de codificadores controláveis isomorfos a partir das entradas e dos estados) : Dado $X_{\oplus} Q$, seja $Y$ qualquer grupo isomorfo a $X_{\ominus} Q$ via $\beta$, isto é, $Y \stackrel{\beta}{\cong} X_{\oplus} Q$. Seja $m \in \mathbb{N}$ definido por $m=\frac{|Q|}{2}$ se $|Q|$ é par e $m=\frac{|Q|+1}{2}$ se $|Q|$ é ímpar. Se $H_{0}$ e $H_{1}$, definidos respectivamente por (7) e (8), são tais que $H_{0} \neq H_{1}$, e se existir um homomorfismo sobrejetor $\delta: X_{\oplus Q} \rightarrow Q$ tal que

$$
\begin{aligned}
& \text { i) } \operatorname{Ker}(\delta)=H_{1} \\
& \text { ii) }\left|\left\{\delta_{m}\left(\stackrel{(m)}{x}, e_{Q}\right): \stackrel{(m)}{x} \in X^{m}\right\}\right|>m .
\end{aligned}
$$

Então, $M_{\oplus}=(X, Y, Q, \delta, \beta)$ é um codificador isomorfo controlável.

\footnotetext{
${ }^{1}$ Isto $\epsilon$ o princí pio da controlabilidade : $j$-controlabilidade implica $j+1$ controlabilidade.
}

Prova : Pelas hipóteses do teorema é claro que $M_{\odot}=(X, Y, Q, \delta, B)$ é um codificador isomorfo. Só resta então provar a controlabilidade. Para isso, seja $Q_{0 i} O$ subgrupo normal de $Q$ definido no Lema 17. Pelo Teorema 19 , temos que $M_{\circlearrowright}=(X, Y, Q, \delta, \beta)$ é não controlável se e somente se $\left|Q_{0_{i}}\right|<|Q|$, para todo $i \in \mathbb{N}$. Mas, $\left|Q_{0_{i}}\right|<|Q|$ implica que $\left|Q_{0_{i}}\right| \leq \frac{|Q|}{2} \leq m$. Assim, $M_{\ominus}=(X, Y, Q, \delta, \beta)$ é não controlável se, e somente se, $\left|Q_{0 i}\right| \leq m$. Como $\left|Q_{0 m}\right|>m$, então $Q_{0 m}=Q \cdot \log o, M_{\ominus}=(X, Y, Q, \delta, \beta)$ é controlável.

Teorema 21 (Construção de codificadores isomorfos controláveis a partir das saiddas) : Dado um grupo finito $Y$, suponha que $U_{0} \triangleleft Y$ e $U_{1} \triangleleft Y$ são subgrupos normais tais que :

$$
\begin{aligned}
\text { i) } & \left|U_{0}\right|=\left|U_{1}\right| \\
\text { ii) } & U_{1} \neq U_{0} \\
\text { iii) } & \frac{Y}{U_{1}} \cong \frac{Y}{U_{0}} .
\end{aligned}
$$

Então,

$-Y \stackrel{\xi}{\cong} X_{\oplus} Q$, para algum isomorfismo $\xi$.

- Se $H_{0}$ e $H_{1}$ são os subgrupos normais de $X_{\ominus} Q$ tais que $U_{0} \stackrel{\xi}{\cong} H_{0}$ e $U_{1} \stackrel{\xi}{\cong} H_{1}$, então $H_{0}$ satisfaz a equação (7); $H_{1} \in \mathcal{X}$, onde $\mathcal{X}$ é definido por (6). Além disso, $H_{0} \neq H_{1}$.

- Se existir um homomorfismo sobrejetor $\delta: X_{\ominus} Q \rightarrow Q$ tal que

$$
\begin{aligned}
& \text { i) } \operatorname{Ker}(\delta)=H_{1} \\
& \text { ii) }\left|\left\{\delta_{m}\left(\stackrel{(m)}{x}, e_{Q}\right): \stackrel{(m)}{x} \in X^{m}\right\}\right|>m,
\end{aligned}
$$

onde $m$ é definido pelo Teorema 20 e $\beta=\xi^{-1}$. Então, $M_{\ominus}=(X, Y, Q, \delta, \beta)$ é um codificador isomorfo controlável.

\section{Prova :}

- Pelo Teorema 16, Y $\cong U_{0 \ominus \frac{Y}{U_{0}}}$. Para $y \in Y$, seja $y_{1} \in U_{0}$ e seja $y_{2} \in Y$ o representante de $\frac{Y}{U_{0}}$, tal que $y=\left(y_{1}, y_{2}\right)$. Sejam $X, Q$ grupos tais que $U_{0} \stackrel{\theta_{1}}{\cong} X$ e $\frac{Y}{U_{0}} \stackrel{\theta_{2}}{\cong} Q$, para alguns isomorfismos $\theta_{1}$ e $\theta_{2}$. Então, $Y \cong U_{0 \oplus \frac{Y}{U_{0}}} \stackrel{\xi}{\cong} X_{\oplus} Q$, onde $\xi=\left(\theta_{1}, \theta_{2}\right)$;

- Para $y=\left(y_{1}, y_{2}\right) \in Y \cong U_{0 \oplus \frac{Y}{U_{0}}}$, temos que o representante de y em $X_{\oplus} Q$ é $\left(\theta_{1}\left(y_{1}\right), \theta_{2}\left(y_{2}\right)\right)$. Em particular, se $y \in U_{0}$, então $y=\left(y, e_{Y}\right)$, daí $\xi(y)=\xi\left(y, e_{Y}\right)$ $=\left(\theta_{1}(y), \theta_{2}\left(e_{Y}\right)\right)=\left(\theta_{1}(y), e_{Q}\right) \in H_{0} \subset X_{\oplus} Q$. Logo, $H_{0}$ satisfaz a equação (7). Por outro lado, como $\frac{Y}{U_{1}} \cong$ $\frac{Y}{U_{0}} e \frac{Y}{U_{0}} \cong Q$ temos que $U_{1}=K \operatorname{Ker}\left(\phi_{1}\right)$, onde $\phi_{1}$ é algum homomorfismo sobrejetor $\phi_{1}: Y \rightarrow Q$. Considere a composição de homomorfismos $\phi_{10} \xi^{-1}: X_{\oplus} Q \rightarrow Q$, então $\phi_{10} \xi^{-1}$ é sobrejetor e $\operatorname{Ker}\left(\phi_{10} \xi^{-1}\right)=\{(x, q)$ : $\left.\phi_{10} \xi^{-1}(x, q)=e_{Q}\right\}=\left\{(x, q): \phi_{1}\left(\xi^{-1}(x, q)\right)=e_{Q}\right\}$ $=\left\{(x, q): \xi(y)=(x, q), \phi_{1}(y)=e_{Q}\right\}=H_{1}$. Finalmente, fica claro que $U_{0} \neq U_{1}$ implica $H_{0} \neq H_{1}$; 
- Fazendo $\delta=\phi_{10} \xi^{-1}$, as condições do Teorema 20 são satisfeitas.

Por outro lado, em [11] é provado que se a seção de treliça for isomorfa a um grupo abeliano cíclico, isto é, $Y \approx \mathbb{Z}_{n}$, então o correspondente codificador é não controlável. Com isso, somente os grupos abelianos não cíclicos é que poderão levar a bons códigos convolucionais abelianos.

Tendo como base o Teorema 21, iremos considerar o caso de códigos convolucionais abelianos cujos elementos do grupo das entradas são $k$-uplas binárias, o grupo dos estados são $(n-k)$-uplas binárias, e consequentemente o grupo das saídas são $n$-uplas binárias, mais especificamente $Y \approx$ $\mathbb{Z}_{2}^{n} \approx \mathbb{Z}_{2}^{k} \oplus \mathbb{Z}_{2}^{n-k}$. A seguir, apresentamos um algoritmo para a construção da máquina $M_{\oplus}=\left(\mathbb{Z}_{2}^{k}, \mathbb{Z}_{2}^{n}, \mathbb{Z}_{2}^{n-k} ; \delta, \beta\right)$ associada a um código convolucional binário de taxa $r=k / n \mathrm{e}$ memória $m=n-k$.

\section{Algoritmo}

Passo 1 - Encontrar $U_{0} \triangleleft \mathbb{Z}_{2}^{n}$ e $U_{1} \triangleleft \mathbb{Z}_{2}^{n}$ tal que, Ia $\left|U_{0}\right|=\left|U_{1}\right|=2^{k}$.

$I b U_{0} \neq U_{1}$.

Ic Se os pesos de Hamming de $U_{0}$ e de $U_{1}, w\left(U_{0}\right)$ e $w\left(U_{1}\right)$, respectivamente, são $w\left(U_{0}\right)=d_{0}$ e $w\left(U_{1}\right)=d_{1}$, então $d_{0}+$ $d_{1}=d$, deve ser tal que

$$
d=\max \left\{w\left(V_{0}\right)+w\left(V_{1}\right)\right\},
$$

$V_{0}$ e $V_{1}$ estão sujeitos a $V_{0}, V_{1} \triangleleft \mathbb{Z}_{2}^{n} ;\left|V_{0}\right|=\left|V_{1}\right|=2^{k}$ e $V_{0} \neq V_{1}$.

Id De modo a evitar transições paralelas, então $U_{0} \cap U_{1}=$ $0 \in \mathbb{Z}_{2}^{n}$.

Passo 2 - Considere $U_{0}$ e $U_{1}$ como subespaços vetoriais de $\mathbb{Z}_{2}^{n}$, e considere as bases $\left\{u_{1}, \ldots, u_{k}\right\}$ de $U_{0}, e$ $\left\{u_{1}, \ldots, u_{t}, v_{1}, \ldots, v_{k-s}\right\}$ de $U_{1}$. Note que $s=0$ se, e somente se, $U_{0} \cap U_{1}=0 \in \mathbb{Z}_{2}^{n}$.

Passo 3 - Seja $\left\{e_{i}\right\}_{i=1}^{n}$ a base canônica de $\mathbb{Z}_{2}^{n}$, onde $e_{i} \hat{e}$ definida por $e_{i}=\left(x_{1}, \ldots, x_{i}\right.$,

$\left.\ldots, x_{n}\right)$ e seus componentes são tais que $x_{j}=0$ if $j \neq i$ $e x_{j}=1$ se $j=i$. Então, defina o mapeamento de rotulamentos como sendo o isomorfismo $\beta: \mathbb{Z}_{2 \oplus}^{k} \mathbb{Z}_{2}^{n-k} \rightarrow \mathbb{Z}_{2}^{n}$ tal que

$$
\begin{aligned}
& \beta^{-1}\left(u_{i}\right)=e_{i}, \\
& \beta^{-1}\left(v_{j}\right)=e_{k+j} ;
\end{aligned}
$$

com a finalidade de otimizar a distância livre, a definição de $\beta$ para os restantes $n-(2 k-s)$ vetores da base $\left\{e_{i}\right\}_{i=1}^{n} \dot{e}$ deixada para o Passo 7.

Passo 4 - Calcular $H_{0}=\beta^{-1}\left(U_{0}\right), H_{1}=\beta^{-1}\left(U_{1}\right), e$ $\Pi_{2}\left(H_{1}\right)$, onde $\Pi_{2}: \mathbb{Z}_{2}^{n} \rightarrow \mathbb{Z}_{2}^{n-k}$ é a projeção definida por $\Pi_{2}\left(x_{1}, \ldots, x_{k}, x_{k+1}, \ldots, x_{n}\right)=\left(x_{k+1}, \ldots, x_{n}\right)$.

Passo 5 - Usando os elementos do grupo das classes laterais $\frac{Z_{2}^{n}}{H_{1}}$, defina a aplicação do próximo estado como sendo o homomorfismo de grupos $\delta: \mathbb{Z}_{2}^{n} \rightarrow \mathbb{Z}_{2}^{n-k}$ com as seguintes condições

5a $\operatorname{Ker}(\delta)=H_{1}$, significando que

$$
\delta\left(e_{k+j}\right)=0 \text { paraj }=1, \ldots, k-s .
$$

5b Se $n-(2 k-s)>0$ então, $\delta\left(H_{0}\right) \cap \Pi_{2}\left(H_{1}\right)=0 \in$ $\mathbb{Z}_{2}^{n-k}$, significando que

$$
\delta\left(e_{i}\right) \notin \Pi_{2}\left(H_{1}\right), \text { para } i=1, \ldots, k .
$$

Passo 6 - A definição de $\delta$ para os remanescentes $n-(2 k-$ s) vetores da base $\left\{e_{i}\right\}_{i=1}^{n}$ deve ser feito de modo que,

6a Satisfaça 0 teste de controlabilidade

$\left|\left\{\delta_{2^{n-k-1}}\left(\begin{array}{c}\left(2^{n-k-1}\right) \\ x\end{array}, 00\right):{ }^{\left(2^{n-k-1}\right)} x \in \mathbb{Z}_{2}^{k \cdot 2^{n-k-1}}\right\}\right|$

$>2^{n-k-1}$.

6b Tratando de que $\delta\left(x, \delta\left(H_{0}\right)\right) \notin \Pi_{2}\left(H_{1}\right)$ para todo $x \in$ $X$.

6c Se $\delta\left(x, \delta\left(H_{0}\right)\right) \notin \Pi_{2}\left(H_{1}\right)$ para todo $x \in X$ então, fazer outra tentativa para que $\delta\left(x_{2}, \delta\left(x_{1}, \delta\left(H_{0}\right)\right)\right) \notin \Pi_{2}\left(H_{1}\right)$. para todo $x_{2}, x_{1} \in X$. Tentar outra vez para $x_{3} \in X$, e assim por diante. Usando a definição de $\delta$ com relação à base $\left\{e_{i}\right\}_{i=1}^{n}$, escrever a regra explícita de $\delta$.

Passo 7 - Defina $\beta$ para os remanescentes $n-(2 k-s)$ vetores da base $\left\{e_{i}\right\}_{i=1}^{n}$ fazendo uma adequada distribuiç̧ão dos pesos conforme a dinâmica da treliça obtida através de $\delta$ no Passo 5 e Passo 6. Usando a definição de $\beta$ em relação $\grave{a}$ base $\left\{e_{i}\right\}_{i=1}^{n}$, escrever a regra explicita de $\beta$.

Este algoritmo gera a máquina $M_{\oplus}=$ $\left(\mathbb{Z}_{2}^{k}, \mathbb{Z}_{2}^{n}, \mathbb{Z}_{2}^{n-k}, \delta, \beta\right)$ associada a um código convolucional binário, não catastrófico com taxa $r=k / n$, memória $m=n-k$ e $d_{\text {free }} \geq d$.

Exemplo 22 Construção de um código convolucional binário a partir da máquina $M_{\ominus}=\left(\mathbb{Z}_{2}^{2}, \mathbb{Z}_{2}^{4}, \mathbb{Z}_{2}^{2}, \delta, \beta\right)$

Passo I - Determinação de $U_{0}$ e $U_{1}$,

$$
U_{0}=\{0000,1001,1110,0111\} \quad U_{1}=\{0000,1100,0011,1111\}
$$

Então, os pesos de Hamming de $U_{0}$ e de $U_{1}$ são $w\left(U_{0}\right)=$ $w\left(U_{1}\right)=2$, respectivamente.

Passo 2 - Determinação das bases para $U_{0}$ e para $U_{1}$ : $\{1001,1110\}$ é uma base para $U_{0}, e\{1100,0011\}$ é uma base para $U_{1}$. Note que, como $U_{0} \cap U_{1}=\{0000\}$, então $s=0$.

Passo 3 -

$$
\begin{array}{ll}
\beta^{-1}(1001)=1000 & \beta^{-1}(0011)=0010 \\
\beta^{-1}(1110)=0100 & \beta^{-1}(1100)=0001
\end{array}
$$

Passo 4 -

$$
\begin{gathered}
H_{0}=\{(00,00),(10,00),(01,00),(11,00)\} \\
H_{1}=\{(00,00),(00,10),(00,01),(00,11)\} \\
\Pi_{2}\left(H_{1}\right)=\{00,10,01,11\}
\end{gathered}
$$

Passo 5 - Para que $\operatorname{Ker}(\delta)=H_{1}$, temos

$$
\begin{aligned}
& \delta(00,10)=00 \\
& \delta(00,01)=00 .
\end{aligned}
$$

Como $n-(2 k-s)=4-(4-0)=0$ então, não é possivel que $\delta\left(H_{0}\right) \cap \Pi_{2}\left(H_{1}\right)=\{00\}$. Logo,

$$
\begin{aligned}
& \delta(10,00)=11 \\
& \delta(01,00)=01
\end{aligned}
$$




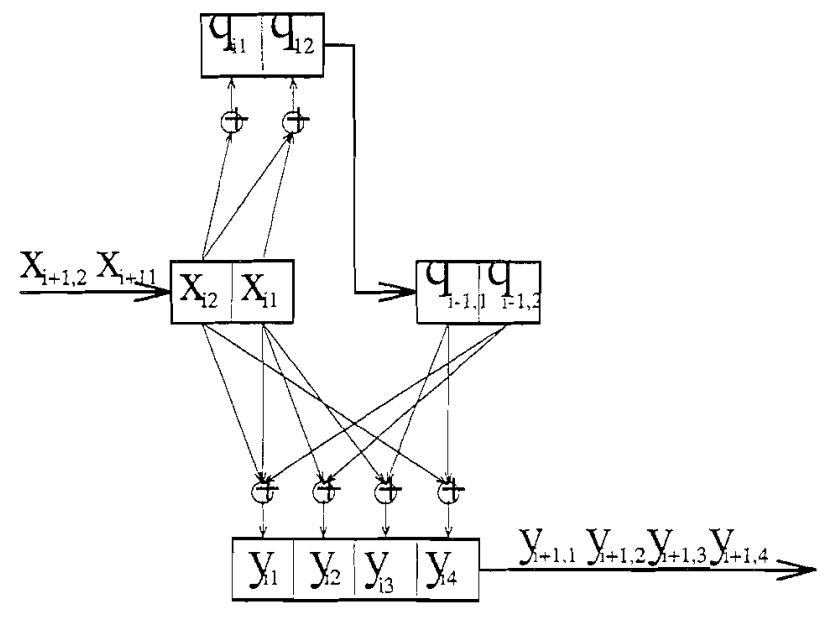

Figura 5. Codificador convolucional abeliano $M_{\oplus}=$ $\left(\mathbb{Z}_{2}^{k}, \mathbb{Z}_{2}^{n}, \mathbb{Z}_{2}^{n-k}, \delta, \beta\right)$.

Passo 6 -Neste exemplo não existem $n-(2 k-s)$ vetores remanescentes $\left\{e_{i}\right\}_{i=1}^{4}$, pois $n-(2 k-s)=4-(4-0)=0$. Portanto, $\delta$ é dado por

$$
\begin{aligned}
& \delta\left(x_{2}, x_{1} ; q_{1}, q_{2}\right)=x_{2} \delta\left(e_{1}\right)+x_{1} \delta\left(e_{2}\right)+q_{1} \delta\left(e_{3}\right)+q_{2} \delta\left(e_{4}\right) \\
& =x_{2} .11+x_{1} .01+q_{1} .00+q_{2} .00 \\
& =\left(x_{2}, x_{2}+x_{1}\right)
\end{aligned}
$$

Passo 7 - Como não há vetores remanescentes, resta escrever a regra de correspondência de $\beta$, isto é,

$$
\begin{aligned}
& \left.\therefore \bar{w}_{2}, \vec{x}_{1} ; q_{1}, q_{2}\right)=x_{2} \beta\left(e_{1}\right)+x_{1} \beta\left(e_{2}\right)+q_{1} \beta\left(e_{3}\right)+q_{2} \beta\left(e_{4}\right) \\
& =x_{2} .1001+x_{1} .1110+q_{1} .0011+q_{2} .1100 \\
& =\left(x_{2}+x_{1}+q_{2}, x_{1}+q_{2}, x_{1}+q_{1}, x_{2}+q_{1}\right)
\end{aligned}
$$

O codificador resultante é mostrado na Figura 5.. O código correspondente têm taxa $R_{c}=\frac{1}{2}$, distância livre $d_{\text {free }}=5 e$ um ganho assintótico de $3.98 \mathrm{~dB}$.

\section{CONCLUSÕES}

Neste trabalho foram estabelecidas as condições necessárias e suficientes para a construção de códigos convolucionais abelianos. Mostramos que tais códigos são controláveis, completos e mínimos, esta última proposição via uma proposta de redução de estados, e tendo como subclasse os códigos convolucionais elementares. Como consequência do fato de que os códigos convolucionais abelianos cuja seção de treliça é isomorfa a um grupo cíclico implica em o correspondente codificador ser não controlável, então um algoritmo de construção de códigos convolucionais abelianos tendo como grupo das entradas, de estados e de saídas, $k$ uplas, $(n-k)$-uplas e $n$-uplas binárias, respectivamente, foi proposto. Exemplos de codificadores convolucionais elementares e abelianos foram apresentados.

\section{REFERÊNCIAS}

[1] M.D.Trott, The Algebraic Structure of Trellis Codes, Ph.D. Dissertation, Dept. of Elect. Eng., Stanford University, Stanford, CA, Aug. 1992.

[2] H.A.Loeliger, On Euclidean-Space Group Codes, Ph.D. Dissertation, Swiss Federal Institute of Technology, Zurich, 1992.

[3] G. D. Forney, "Geometrically uniform codes," IEEE Trans. Inform. Theory, vol. IT-37 No 5, pp. 1241-1260, 1991.

[4] J.C.Willems, "Models for dynamics," Dynamics Technical Report, vol. 2, U.Kirchgraber e H.O.Walther, Eds. Wiley and Teubner, 1989.

[5] M.A. Arbib, "Automaton Decomposition and Semigroup Structure," Algebraic Structure of Machine Languages, M.A. Arbib editor, 1968.

[6] M.Hall, The Theory of Groups, Macmillan, New York, 1961.

[7] J.J. Rotman, An Introduction to the Theory of Groups, Springer-Verlag, Fourth ed., 1995.

[8] H.A. Loeliger, G.D. Forney, T. Mittelholzer, and M.D. Trott, "Minimality and observability of group systems," Linear Algebra and its Applications, vol 205-206, pp 937-963, July 1994.

[9] M.A. Arbib, Brains, Machines, and Mathematics, Springer-Verlag, Second ed. ; 1986.

[10] G.D. Forney and M.D. Trott, "The dynamics of group codes : state spaces, trellis diagrams and canonical encoders", IEEE Trans. Inform. Theory, vol IT 39(5) :1491-1513, September 1993.

[11] J. P. Arpasi, Codificadores Convolucionais Homomorfos, Tese de Doutorado, FEEC-UNICAMP, Junho 1997.

Jorge Pedraza Arpasi nascido em Puno, Peru em 1961. Bacharel em Matemática pela Universidad San Agustin do Peru, 1987. Mestre em Matemática pelo Instituto de Matmática Pura e aplicada (IMPA), 1990. Doutor em Engenharia Elétrica, area de comunicações, 1996. Pos-Doutorado no Departamento de Telemática da Faculdades de Engenharia Elátrica e Computação (FEEC) da Universidade Estudual de Campinas desde 1997 até a presente data. Area de Trabalho: Códigos correctores de errros.

Reginaldo Palazzo JR. received the Electrical Engineering and MSEE degrees from University Estadual de Campinas - UNICAMP, SP, Brazil, in 1975 and 1977 respectively, and the Engineer and PH.D. degrees from the University of California,Los Angeles, CA, USA, in 1981 and 1984, respectively, In May 1985 he joined the Faculty of Eletrical and Computing Engineering, UNICAMP, where he received the Livre-Docência degree in 1987. Since 1996 he is a Professor and Chair of the AIgebraic and Geometric Coding Group. His research interests are in coding, information, and communication theory. 